custom, to be that of its intrusion. We are entitled, however, to consider the previous history of the material composing these intrusive Archæan masses, and, in view of their predominantly banded structure, which marks them off as in some way different from later intrusive masses of similar composition, such consideration seems forced upon us.

Now the banded character of the Archæan gneiss suggests a partial derivation by melting from some stratiform materials such as sedimentary or volcanic rocks, or at any rate from rocks showing marked small-scale differentiation into basic and acid types. I do not think that stratiform differentiation during or previous to crystallisation can be seriously put forward as a cause of the banding, in view of the rarity of this phenomenon in more recent granites, and the fact that in them it is largely a marginal effect.

May we not then have in these Archæan gneisses the recrystallised remnants of still older sediments and lavas, and who is to say that they may not also embrace portions of the original surface on which water first settled, but so obscured by recrystallisation that the question of its molten or planetesimal origin is now unsolvable?

The difference between the two views is simply that one regards the history of sedimentation on the earth as cut off sharply by intrusion, while the other sees it extending still further back into the mists of the past, beyond the point where human vision is any longer capable of discrimination. Where, on either view, is the decisive criterion between the nebular and planetesimal hypotheses?

\section{W. B. WRIGHT}

Manchester, June 27, 1922.

\section{Wegener's Displacement Theory.}

WEGENER's speculations have attracted so much attention that there must be many who would be glad to find some simple means of testing his fittings and coincidences for themselves. Owing to the distortion present in all maps such tests must be carried out on a globe. Wegener himself uses tracing paper, which must be cut and slashed in order that it may even approximately fit the surface ; and any one who has tried it will admit that it is difficult to obtain satisfactory results. An easier plan is to roll out a lump of modelling wax or plasticine into a sheet of moderate thickness. The sheet may then be pressed upon the globe and cut to the required shape. According to my own experience, the best method is to cut the sheet a little smaller than the area that is to be represented, so that the actual margin appears all round it, and to build it outwards to this margin by the addition of small pieces of wax. Old plasticine which has become rather dry works very well and does not stick to the globe.

But much more precise tests can be carried out with the help of some form of triangular compasses. The three points of the compasses may be placed on three critical points of the globe and afterwards transferred, without altering their relative positions, to any other part of the globe that may be desired. The ordinary triangular compasses of the draughtsman are very little use upon a spherical surface, but a fairly convenient instrument can be constructed with an ordinary one-jointed two-foot rule as its basis. A point about an inch long is fixed near the joint, and each arm is provided with a sliding carrier. Each carrier bears a short sleeve through which a pointed rod, such as a knitting needle, slides rather stiffly. These rods form the other two points, and all three should stand approximately at right angles to the plane of the rule.
This is an easily constructed type, but much more convenient forms can be devised. If, for example, the arms are arcs of circles, of suitable diameter, so that they may stand concentric with the globe, the points may all be of fixed length, and the most troublesome of the adjustments required by the straight-armed form will be avoided.

This is not the place to discuss Wegener's views, but the use of triangular compasses seems to show that a rather high degree of plasticity is necessary in the masses of "Sial" in order to produce the coincidences on which he bases his calculation of the probability that his theory is correct.

\section{Sedgwick Museum} Cambridge, June $2 \mathrm{I}$.

PhILIP LAKE.

\section{Opalescence Phenomena in Liquid Mixtures.}

IT is well known that liquids which mix completely above a certain critical temperature, e.g. phenol and water, exhibit a strong and characteristic opalescence as the temperature of the mixture is lowered to a point slightly above that at which the components separate. A quantitative theory of this phenomenon was put forward by Einstein (Annalen der Physik, vol. 33, I9Io) on the basis of thermodynamical reasoning, the spontaneous local fluctuations of concentration of the mixture being taken into account and the light-scattering due to the resulting fluctuations of refractive index being evaluated. He obtained as the expression for the light-scattering

$$
\pi^{2}\left(\mathrm{M} / \mathrm{N} \lambda^{4}\right) \cdot v\left(\frac{\partial \mu^{2}}{\partial \kappa}\right)^{2} / \frac{\partial(\log p)}{\partial \kappa} \text { per unit volume, }
$$

where $\mu$ is the refractive index of the mixture and $\partial(\log p) / \partial \kappa$ expresses the rate of change of the vapour pressure of one of the components with concentration, a quantity which becomes very large as the critical temperature and concentration are approached, thus giving rise to a marked opalescence. It should be pointed out, however, that Einstein's expression does not include the whole effect, for we have also to consider the result of the fluctuation of density of either component taken separately, and to add to Einstein's formula

$\left(\pi^{2} / \mathrm{I} 8\right)\left(\mathrm{RT} / \mathrm{N} \lambda^{4}\right)\left[\beta_{1}\left(\mu_{1}^{2}-\mathrm{I}\right)^{2}\left(\mu_{1}{ }^{2}+2\right)^{2}\right.$

$$
\left.+\beta_{2}\left(\mu_{2}^{2}-I\right)^{2}\left(\mu_{2}^{2}+2\right)^{2}\right]
$$

where $\beta_{1}, \beta_{2}, \mu_{1}, \mu_{2}$ are respectively the compressibilities and refractive indices of the components. Further, the light-scattering due to the anisotropy and arbitrary orientation of the molecules of the components has also to be added.

The result of these corrections of Einstein's investigation may briefly be indicated. Very near the temperature at which the mixture separates into two phases, the fluctuations of concentration contribute by far the larger portion of the effect. But at higher and lower temperatures the effects of fluctuations of density and molecular anisotropy are no longer negligible, and when the temperature is sufficiently removed from the critical point they form a substantial part of the whole. Further, the increase in relative importance of the effect of molecular anisotropy in these circumstances should result in an increase in the proportion of unpolarised light in the transversely-scattered beam as we recede from the critical temperature.

The foregoing indications of theory have been confirmed generally in a series of experiments over a wide range of temperatures on light-scattering in phenol-water mixtures undertaken under the writer's direction by Mr. V. S. Tamma. It is found that the increased opalescence of the mixture over 